EUROPHYSICS LETTERS

Europhys. Lett., (), pp. ()

\title{
Impurity scattering and frequency dependent conductivity in spin density waves
}

\author{
Attila Virosztek ${ }^{1,2}$, Balázs Dóra ${ }^{1}$ and Kazumi Maki ${ }^{3}$ \\ 1 Department of Physics, Technical University of Budapest, H-1521 Budapest, Hungary \\ 2 Research Institute for Solid State Physics and Optics P.O.Box 49, H-1525 Budapest, \\ Hungary \\ 3 Department of Physics and Astronomy, University of Southern California, Los Angeles, \\ CA 90089-0484, USA \\ (received ; accepted ) \\ PACS. 74.70Kn - Organic Superconductors. \\ PACS. 78.20-e - Optical properties of bulk materials and thin films. \\ PACS. 75.30Fv - Spin density waves.
}

\begin{abstract}
The quasiparticle contribution to the frequency dependent electric conductivity in the presence of randomly distributed impurities is calculated within mean field theory for spin density waves as formed in quasi one dimensional conductors like Bechgaard salts. Interchain hopping is taken into account and the effects of imperfect nesting are considered. In case of an electric field perpendicular to the chain direction there is no collective contribution to the conductivity and our results are directly applicable to recent measurements on both $(\mathrm{TMTSF})_{2} \mathrm{PF}_{6}$ and quenched (TMTSF $)_{2} \mathrm{ClO}_{4}$. The experimental data are well described by the theory in its clean limit, with somewhat larger scattering in the $\mathrm{ClO}_{4}$ salt.
\end{abstract}

Spin density wave (SDW) is one of the ground states of quasi one dimensional electronic systems, and commonly found at low temperature in Bechgaard salts (TMTSF) $)_{2} \mathrm{X}$ with $\mathrm{X}=\mathrm{PF}_{6}, \mathrm{ClO}_{4}$, etc [1] 2]. Both the appearance of SDW in Bechgaard salts with the pressure dependence of the SDW transition temperature $T_{c}$, and the electronic properties of the SDW are well described in terms of the standard model, where the approximate nesting of the quasi one dimensional Fermi surface (i.e. the imperfect nesting), and the repulsive Coulomb interaction between electrons are the crucial ingredients[3. In particular, the enhanced gap to $T_{c}$ ratio compared to the weak coupling BCS value is interpreted as the consequence of

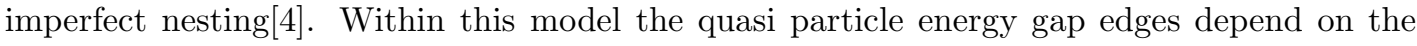
quasi particle momentum perpendicular to the conducting chains (the $\mathbf{b}$ direction) as

$$
\Delta_{ \pm}\left(p_{y}\right)= \pm \Delta+\varepsilon_{0} \cos \left(2 b p_{y}\right)
$$

where $\Delta$ is the order parameter and $\varepsilon_{0}$ is the "unnesting" parameter characterizing the deviation from one dimensionality. Indeed, the above momentum dependent energy gap is inferred from the magnetoresistance data[5] in the SDW state of (TMTSF) ${ }_{2} \mathrm{PF}_{6}$, and it offers

Typeset using EURO- $\mathrm{TE}_{\mathrm{E}} \mathrm{X}$ 
an explanation of the difference between the energy gap as observed by transport and optical measurements for a number of density wave materials [6]. While the optical (pair breaking) gap is still given by $2 \Delta$, dc transport is sensitive to the smaller gap in the density of states 4 .

The frequency dependent electric conductivity of the SDW in Bechgaard salts has been studied over decades[2]. Unfortunately however, the clear understanding of the physical situation still appears to be lacking. Earlier experiments were mostly done in the geometry E $\|$ a, i.e. the electric field parallel to the chain direction[7]. Under these circumstances it is expected that the phason couples strongly to the electromagnetic field in analogy to the mean field treatment of CDW by Lee, Rice and Anderson 8, 9]. In SDW, most of the optical weight is shifted to the phason mode and gives rise to low energy absorption 10 for $\omega \ll 2 \Delta$, since in the absence of pinning the transverse phason is naturally coupled to the transverse phonon[11] as well as the photon. However, in general the phason is pinned by impurities or crystalline defects as evidenced by the existence of the threshold electric field in the non Ohmic dc conductivity in $(\mathrm{TMTSF})_{2} \mathrm{NO}_{3}$ and $(\mathrm{TMTSF})_{2} \mathrm{PF}_{6}[12,13$, and therefore the longitudinal phason gets mixed in as well. On the other hand we have shown[10] that the longitudinal phason is almost completely removed from the low frequency range in an SDW due to the Anderson-Higgs mechanism 14]. We believe that this is the main reason for the lack of a clear optical energy gap in the data taken in the geometry $\mathbf{E} \| \mathbf{a}$, since first of all the optical weight at the gap region $\omega \geq 2 \Delta$ is shifted down to the pinned mode at $\omega \ll 2 \Delta$, which in turn is depleted due to the admixture with the longitudinal phason which is nothing but the plasmon in a SDW.

In this situation the frequency dependent conductivity perpendicular to the chains is ideal to measure the optical energy gap, since in the geometry $\mathbf{E} \perp \mathbf{a}$ the electromagnetic field does not couple to the phason [11]. Within mean field theory we only need to consider the so called quasiparticle contribution to the conductivity since no collective contribution is present in this case. We obtain an expression for the perpendicular electric conductivity which is very similar to the one derived by Mattis and Bardeen 15, and by Abrikosov et.al. 16. for $s$-wave superconductors except one crucial point. The authors of both papers 15, 16 introduced a simplification which is valid either in the anomalous skin limit or in the dirty limit. Unfortunately, for organic conductors and also for high $T_{c}$ cuprate superconductors none of the above approximations applies, since they are usually in the clean limit and the penetration depth of the ac field is much larger than $10 \mu \mathrm{m}$. An expression for the SDW conductivity appropriate for the above conditions has already been obtained in the extreme clean (or collisionless) limit characterized by infinite quasiparticle mean free path 17], but for a more complete description the effect of finite mean free path should certainly be taken into account.

In this report we incorporate the quasiparticle mean free path in terms of impurity scattering, characterized by a forward $\left(\Gamma_{1}\right)$ and backscattering $\left(\Gamma_{2}\right)$ rate 18 . These two rates stand for the amplitude of scattering processes involving electrons originating from and arriving to the same or different Fermi sheets of the quasi one dimensional Fermi surface respectively. Then we compare the theoretical results with recent experimental data on the SDW of $(\mathrm{TMTSF})_{2} \mathrm{PF}_{6}$ and $(\mathrm{TMTSF})_{2} \mathrm{ClO}_{4}$ 19, 20]. The agreement looks rather reasonable as long as we use $2 \Delta(T=0) \approx 70 \mathrm{~cm}^{-1} \approx 100 \mathrm{~K}$ for both compound, where $\Delta(T=0)$ is the SDW order parameter at zero temperature. The order parameter thus determined appears to be about a factor two larger than the ones determined from transport measurements, for example $\Delta(T=0)=21 \mathrm{~K}$ was deduced in Ref. [5]. This discrepancy however may be understood by invoking the effect of imperfect nesting [6] expressed by Eq.(11). On the other hand, the SDW transition temperature $T_{c}$ can be reduced from its weak coupling BCS value of $28 \mathrm{~K}$ for at least two reasons. First is the effect of imperfect nesting on $T_{c}$ which is still within the realm 
of mean field theory, and is suggested in particular by the fact that the same energy gap has been observed in both (TMTSF) ${ }_{2} \mathrm{PF}_{6}$ and (TMTSF $)_{2} \mathrm{ClO}_{4}$. Second is the suppressing effect of fluctuations, although in our case they should be at least two dimensional. Indeed, the observed frequency dependence of the conductivity just above $T_{c}$ in Bechgaard salts 20 suggests strongly the pseudogap phenomenon.

Our calculation of the frequency dependent conductivity involves standard diagrammatic treatment of impurity scattering in density waves 18. In order to evaluate the quasiparticle contribution we dress a single loop of current correlation function by impurity lines. Self energy corrections are taken into account in the non crossing approximation, while vertex corrections will vanish due to the constant scattering rates $\Gamma_{1}$ and $\Gamma_{2}$, and to the structure of the transverse current

$$
v_{y}(\mathbf{p})=\sqrt{2} v_{y} \sin \left(b p_{y}\right),
$$

where $v_{y}=\sqrt{2} b t_{b}$ is the relevant electron velocity perpendicular to the chains and $t_{b}$ is the hopping integral in the $\mathbf{b}$ direction. The thermal product corresponding to the current correlation function is written down, and analytic continuation to the real frequency axis leads directly to $\sigma_{y y}(\omega)$. For simplicity, we cite here only the results for perfect nesting, leaving the detailed treatment of imperfect nesting effects to a forthcoming publication. The frequency dependent conductivity of a SDW in the $\mathbf{b}$ direction consists of two contributions:

$$
\operatorname{Re}\left[\sigma_{y y}(\omega)\right]=e^{2} v_{y}^{2} N_{0} \frac{\operatorname{Im}\left[I_{n}(\omega)+I_{p}(\omega)\right]}{\omega},
$$

where $N_{0}$ is the total density of states (including spin degeneracy) of the electron system, and the "normal" contribution (involving intraband processes) is given by

$$
\operatorname{Im} I_{n}(\omega)=\int_{G}^{\infty} \frac{d E}{2}\left[\tanh \frac{E+\omega}{2 T}-\tanh \frac{E}{2 T}\right] \operatorname{Re}\left\{F[u(E+\omega), u(E)]-F\left[u(E+\omega), u^{*}(E)\right]\right\},
$$

while the so called pair breaking (interband) contribution is present only for frequencies larger than the gap $2 G$ in the quasiparticle spectrum:

$$
\operatorname{Im} I_{p}(\omega>2 G)=\int_{G}^{\omega-G} \frac{d E}{2} \tanh \frac{\omega-E}{2 T} \operatorname{Re}\left\{F\left[u(\omega-E),-u^{*}(E)\right]-F[u(\omega-E),-u(E)]\right\} .
$$

In the above two expressions the function $F$ of two variables is given by

$$
F\left(u, u^{\prime}\right)=\frac{-\left[1-\frac{1-u u^{\prime}}{\sqrt{1-u^{2}} \sqrt{1-\left(u^{\prime}\right)^{2}}}\right]}{\Delta\left[\sqrt{1-u^{2}}+\sqrt{1-\left(u^{\prime}\right)^{2}}\right]-\Gamma_{1}},
$$

and the function $u(E)$ is defined by

$$
\frac{E}{\Delta}=u\left(1-\frac{\alpha}{\sqrt{1-u^{2}}}\right)
$$

Here the pair breaking parameter $\alpha=\Gamma / \Delta$ with $\Gamma=\Gamma_{1}+\frac{1}{2} \Gamma_{2}$, while the gap is related to the order parameter as $G=\Delta u_{0}^{3}$ where $u_{0}^{2}=1-\alpha^{2 / 3}$, unless we are in the gapless regime very close to $T_{c} 18$.

We note here, that the calculation of the quasiparticle term for the conductivity in the chain direction proceeds rather similarly, except for the following two differences: in order to obtain 


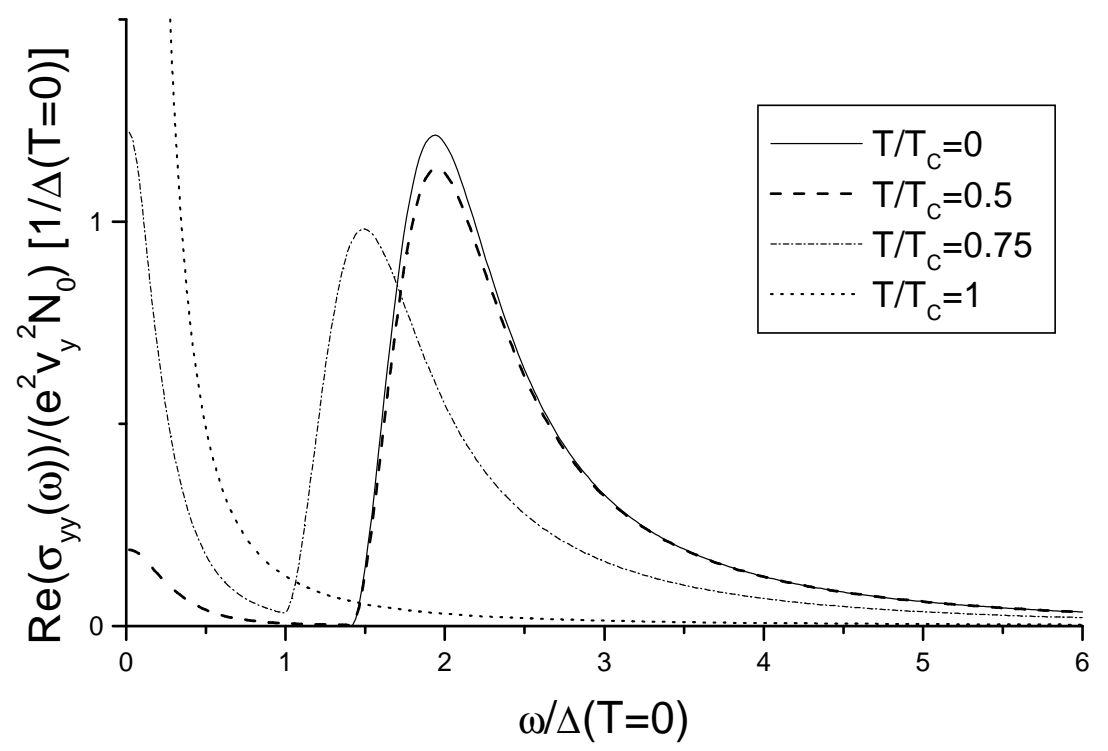

Fig. 1. - Real part of the normalized frequency dependent conductivity in the direction perpendicular to the chains for several temperatures relative to the critical temperature of the spin-density wave transition $T_{c}$. The electron forward- and backscattering rates due to impurities are $\Gamma_{1}=2 \Gamma_{2}=0.08 \Delta(T=0)$, corresponding to a pair breaking parameter $\alpha=0.1$ at zero temperature.

$\operatorname{Re}\left[\sigma_{x x}(\omega)\right]$ we first need to replace $v_{y}$ by the Fermi velocity $v_{F}$ in Eq.(3), and second, because of the important vertex corrections in this case we need to use the following $F$ function

$$
F_{x}\left(u, u^{\prime}\right)=\frac{-\left[1-\frac{1-u u^{\prime}}{\sqrt{1-u^{2}} \sqrt{1-\left(u^{\prime}\right)^{2}}}\right]}{\Delta\left[\sqrt{1-u^{2}}+\sqrt{1-\left(u^{\prime}\right)^{2}}\right]-\left(\Gamma_{1}-\frac{1}{2} \Gamma_{2}\right)\left[1-\frac{1-u u^{\prime}}{\sqrt{1-u^{2}} \sqrt{1-\left(u^{\prime}\right)^{2}}}\right]}
$$

in place of Eq.(6).

Limiting ourselves to the case $\mathbf{E} \perp \mathbf{a}$ (i.e. we use Eq.(6) for the $F$ function), we have evaluated the real part of the conductivity as a function of frequency as given by Eq.(3) for $T / T_{c}=0,0.5,0.75$ and 1 , for two sets of values of the impurity scattering rates leading to $\alpha=0.1$ and 0.25 at zero temperature. The results are shown in Fig. 1 and Fig.2 respectively. It is demonstrated that the normal state $\left(T / T_{c}=1\right)$ Drude peak given by Eq.(3) evaluated for $\Delta=0$ :

$$
\operatorname{Re}\left[\sigma_{y y}(\omega)\right]=e^{2} v_{y}^{2} N_{0} \frac{\Gamma_{1}+\Gamma_{2}}{\omega^{2}+\left(\Gamma_{1}+\Gamma_{2}\right)^{2}}
$$




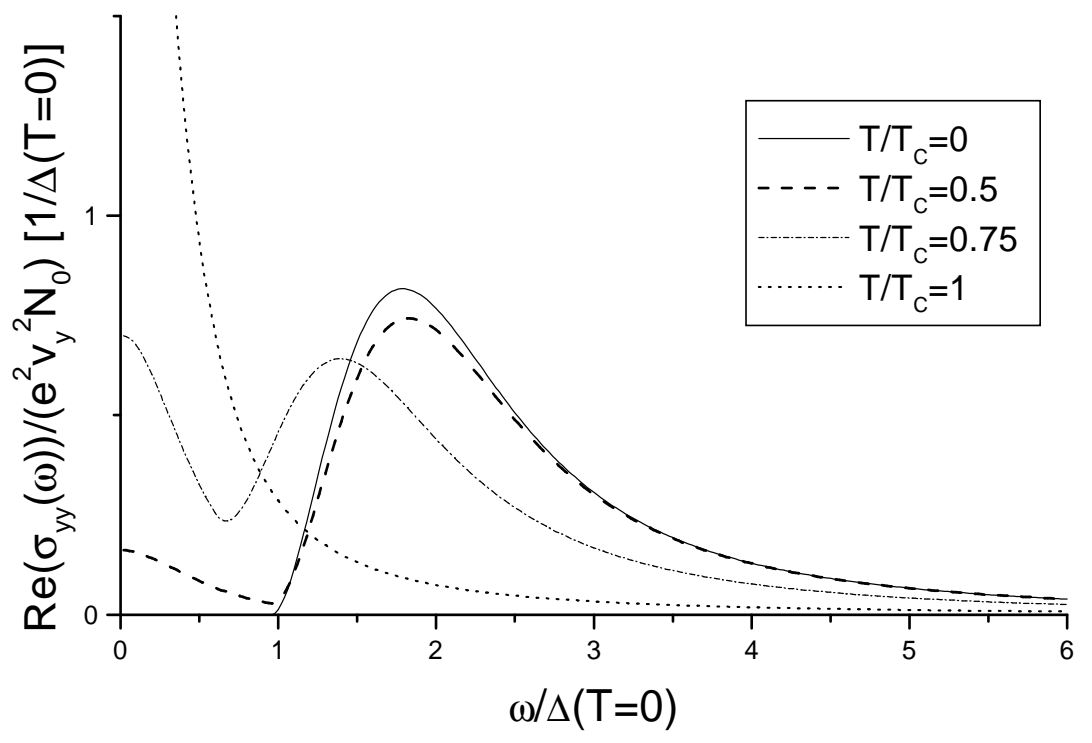

Fig. 2. - Same as in Fig.1, except that here $\Gamma_{1}=2 \Gamma_{2}=0.2 \Delta(T=0)$, corresponding to a zero temperature value of $\alpha=0.25$.

is gradually frozen out as the temperature is lowered, transferring all the spectral weight to the pair breaking peak around $2 \Delta$ at zero temperature. The values of the impurity scattering rates were chosen so that our figures would be representative of the clean limit situation, and would most closely resemble the width and shape of the experimental data for (TMTSF ${ }_{2} \mathrm{PF}_{6}$ and (TMTSF $)_{2} \mathrm{ClO}_{4}$. Unfortunately the detailed quantitative comparison with experiments is hindered by contributions to the frequency dependent conductivity not related to the SDW discussed here. However, we can estimate for example the ratio of the peak width (at half maximum) to the peak position (which is 0.45 on Fig.1) at low temperature, and obtain 0.45 for the $\mathrm{PF}_{6}$ and 0.50 for the $\mathrm{ClO}_{4}$ salt. Our results confirm that these Bechgaard salts are indeed in the relatively clean limit. We note here in passing that if the current flows in the chain direction $(\mathbf{E} \| \mathbf{a})$, than the transport lifetime $\Gamma_{1}+\Gamma_{2}$ in Eq.(9) gets replaced by $2 \Gamma_{2}$ due to the vertex corrections, since only backscattering can cause current damping in the chain direction.

Perhaps some additional analytic results derivable from the previous equations in the experimentally relevant clean limit and at low temperatures are of interest. First we can evaluate the dc conductivity as

$$
\sigma_{i i}^{d c}=e^{2} v_{i}^{2} N_{0} \frac{2 T}{3 \Delta \Gamma u_{0}} e^{-G / T}
$$

where $i=x, y$ and $v_{x}=v_{F}$. It is worth to note that the ratio of the dc resistivities in the two 
directions $v_{y}^{2} \rho_{y y} / v_{F}^{2} \rho_{x x}$ is unity for zero temperature, while takes on the value $\frac{1}{2}\left(1+\Gamma_{1} / \Gamma_{2}\right)$ for $T \rightarrow T_{c}$, indicating a temperature dependence of this ratio even though the scattering rates are themselves temperature independent. Also the shape of the central peak which freezes out for $T \rightarrow 0$ turns out to be different from the usual Lorentzian. If the scattering is not too small, the conductivity exhibits an unconventional $\omega^{-1 / 2}$ behavior in the frequency range $T \ll \omega \ll G$ :

$$
\operatorname{Re}\left[\sigma_{i i}(\omega)\right]=\sigma_{i i}^{d c} \sqrt{\frac{\pi T}{4 \omega}}
$$

Finally, we can evaluate the pair breaking contribution near the threshold $\omega \simeq 2 G$ :

$$
\frac{\operatorname{Re}\left[\sigma_{y y}(\omega)\right]}{e^{2} v_{y}^{2} N_{0}}=\frac{8}{9 \alpha^{2 / 3}} \frac{(\omega-2 G)^{2}}{\Gamma G \omega}
$$

and

$$
\frac{\operatorname{Re}\left[\sigma_{x x}(\omega)\right]}{e^{2} v_{F}^{2} N_{0}}=\frac{1+5 \Gamma_{2} / \Gamma_{1}+\left(3 \Gamma_{2} / 2 \Gamma_{1}\right)^{2}}{36 \alpha^{2 / 3}} \frac{(\omega-2 G)^{2}}{\Gamma_{1} G \omega}
$$

In summary, we have obtained the expression for the frequency dependent conductivity in a SDW for $\mathbf{E} \perp$ a within mean field theory. The effect of the quasiparticle lifetime is incorporated in terms of impurity scattering. The present results are shown on Figs. 1 and 2 in the weak scattering limit, and appear to describe the observed conductivity of both (TMTSF) ${ }_{2} \mathrm{PF}_{6}$ and (TMTSF $)_{2} \mathrm{ClO}_{4}$ reasonably well. It is highly desirable to test how the Drude-like peak disappears at low temperatures. Also the present model highlights the different transport lifetimes for $\mathbf{E} \| \mathbf{a}$ and $\mathbf{E} \perp \mathbf{a}$, which is indeed consistent with some of the magnetotransport measurements [5].

This work was supported by the Hungarian National Research Fund under grant numbers OTKA T020030 and T015552, and by the Ministry of Education under grant number FKFP $0029 / 1999$. One of us (A. V.) acknowledges greatfully the hospitality during his stay at the University of Southern California.

\section{REFERENCES}

[1] Ishiguro T. and Yamaji K., Organic Superconductors, (Springer, Berlin) 1990.

[2] Grüner G., Rev. Mod. Phys., 66 (1994) 1.

[3] Yamaji K., J. Phys. Soc. Jpn., 51 (1982) 2987; ibid. 52 (1983) 1361.

[4] Huang X. and Maki K., Phys. Rev. B, 42 (1990) 6489; Synth. Metals, 55-57 (1993) 2767.

[5] Korin-Hamzić B., Basletić M. and Maki K., Europhys. Lett., 43 (1998) 450.

[6] Mihály G., Virosztek A. and Grüner G., Phys. Rev. B, 55 (1997) R13456.

[7] Schwartz A., Dressel M., Grüner G., Vescoli V., Degiorgi L. and Giamarchi T., Phys. Rev. B, 58 (1998) 1261.

[8] Lee P. A., Rice T. M. and Anderson P. W., Sol. State Commun., 14 (1974) 703.

[9] Virosztek A. and Maki K., Phys. Rev. B, 48 (1993) 1368.

[10] Virosztek A. and Maki K., Phys. Rev. B, 49 (1994) 6074.

[11] Virosztek A. and Maki K., Phys. Rev. B, 53 (1996) 3741.

[12] Tomić S., Cooper J. R., Jérome D. and Bechgandd K., Phys. Rev. Lett., 62 (1989) 462.

[13] Tomić S., Cooper J. R., Kang W., Jérome D. and Maki K., J. Phys. (Paris) I., 1 (1991) 1603. 
[14] Anderson P. W., Phys. Rev., 112 (1958) 1900.

[15] Mattis D. C. and Bardeen J., Phys. Rev., 111 (1958) 412.

[16] Abrikosov A. A., Gor'kov L. P. and Khalatnikov I. M., Sov. Phys. JeTP, 8 (1959) 182.

[17] Virosztek A. and Maki K., Phys. Rev. B, 37 (1988) 2028; Maki K. and Virosztek A., Phys. Rev. B, 41 (1990) 557.

[18] Maki K. and Virosztek A., Phys. Rev. B, 39 (1989) 2511.

[19] Dressel M., Degiorgi L., Brinckmann J., Schwartz A. and Grüner G., Physica B, 230232 (1997) 1008.

[20] Vescoli V., Degiorgi L., Dressel M., Schwartz A., Henderson W., Alavi B., Grüner G., Brinckmann J. and Virosztek A., Phys. Rev. B, (submitted). 